\title{
Crosstalk of Hyperglycemia and Dyslipidemia in Diabetic Kidney Disease
}

\author{
Wen Su ${ }^{a}$ Rong Cao ${ }^{b}$ Yong Cheng He ${ }^{b}$ You Fei Guan ${ }^{c}$ Xiong Zhong Ruan ${ }^{d}$ \\ ${ }^{a}$ AstraZeneca - Shenzhen University Joint Institute of Nephrology, Center for Nephrology and Urology, Department \\ of Physiology, Shenzhen University Health Science Center, Shenzhen University, and b Department of Nephrology, \\ The First Affiliated Hospital of Shenzhen University, Shenzhen, and ' Advanced Institute for Medical Sciences, Dalian \\ Medical University, Dalian, China; ${ }^{\mathrm{d} J o h n}$ Moorhead Research Laboratory, Centre for Nephrology, University College \\ London Medical School, Royal Free Campus, London, UK
}

\section{Keywords}

Diabetic kidney disease - Dyslipidemia - Hyperglycemia . Nuclear receptors $\cdot$ Renal lipid metabolism

\footnotetext{
Abstract

Background: Diabetic kidney disease (DKD) is defined by the functional, structural, and clinical abnormalities of the kidney that are caused by diabetes. Summary: One-third of both type 1 diabetes and type 2 diabetes patients suffer from DKD, which is the leading cause of end-stage renal disease, and is also associated with cardiovascular disease and high public health care costs. Serum glucose level and lipid level are key factors in the pathogenesis of DKD and are modifiable. The goal of this review is to provide an update on the roles of glucose and lipid metabolism in DKD and their crosstalk at the molecular level. We will further discuss the recent advances regarding metabolic nuclear receptors in glucoselipid crosstalk, which may provide new potential therapeutic targets for DKD. Key Message: AMPK, SREBP-1, and some metabolic hormone receptors including liver $\mathrm{X}$ receptors, farnesoid $X$ receptors, and peroxisome proliferator-activated receptors mediate the crosstalk of hyperglycemia and dyslipidemia in diabetic kidney disease and might be potential treatment candidates.

(c) 2017 S. Karger AG, Basel
}

\section{Introduction}

Diabetic kidney disease (DKD), which also known as diabetic nephropathy, is defined as diabetes with albuminuria (ratio of urine albumin to creatinine $\geq 30 \mathrm{mg} / \mathrm{g}$ ) or an impaired glomerular filtration rate (GFR) $(<60 \mathrm{~mL} /$ $\left.\min / 1.73 \mathrm{~m}^{2}\right)$, or both [1]. DKD is the most common complication of diabetes mellitus, and is a leading cause of end-stage renal disease, necessitating dialysis or transplantation. The number of patients with DKD continues to increase despite improved management of diabetes [2, 3]. DKD is characterized by a reduced GFR, glomerular hypertrophy, albuminuria, glomerular basement membrane thickening, mesangial expansion, podocyte loss, glomerular sclerosis, and interstitial fibrosis [4]. The mechanisms responsible for the development and progression of DKD remain largely unknown. In spite of glycemic control and antagonists of the renin-aldosterone system, DKD still progresses in most patients. Apart from genetics, inadequate metabolic control over many years might lead to DKD via epigenetic modification as a cumulative result. Among these metabolic dysregulations, hyperglycemia and dyslipidemia are two key metabolic disorders that have a close association with DKD.

Wen Su and Rong Cao contributed equally to this work.

\section{KARGER}

(C) 2017 S. Karger AG, Basel 
Dyslipidemia is associated with DKD and has been shown to play crucial roles in the development and progression of DKD [5]. Treating uncontrolled glucose levels can directly improve lipid levels, especially those of triglycerides (TG). The most used antidiabetic drug, metformin, exerts a strong glucose-lowering effect, and it can improve the lipid profile at the same time, suggesting there is crosstalk between hyperglycemia and dyslipidemia. However, several glucose-lowering agents do not have a beneficial effect on the lipid profile and even cause weight gain while improving glucose levels. Therefore, it is important to figure out what the background mechanism linking glucose and lipid metabolism is.

The conception "metabolic memory" means the phenomenon where periods of good/bad metabolic control can have long-lasting effects on the development of complications in diabetes even after abolishment of good/bad control in diabetic patients [6]. Clinical observational studies revealed that this metabolic memory could even last more than 25 years, suggesting a long-term environmental programming effect during DKD development [6]. Metabolic nuclear receptors, defined as a group of nuclear hormone receptor transcription factors, play key roles in regulating a variety of metabolic processes such as adipogenesis, glucose metabolism, lipid metabolism, energy metabolism, insulin sensitivity, and so on [7]. Well-known metabolic nuclear receptors include peroxisome proliferator-activated receptors (PPARs), liver X receptors (LXRs), and farnesoid X receptors (FXRs). Dysregulation of theses metabolic nuclear receptors is closely related to metabolic syndrome, and thus plays an important role in the development of DKD.

In this review, we will review the recent progress in elucidating the roles of hyperglycemia and dyslipidemia in DKD and also discuss the roles of metabolic nuclear receptors in glucose-lipid crosstalk.

\section{Hyperglycemia and DKD}

Chronic hyperglycemia plays a major role in the pathogenesis of DKD-related complications. The human kidney is involved in the regulation of glucose homeostasis and abnormalities through the release, uptake, and reabsorption of glucose. The liver and kidney are the only organs in humans that have abundant glucose-6-phosphatase levels and therefore are able to perform gluconeogenesis. Under the fast condition, the liver can produce glucose through glycogenolysis; the kidney, however, cannot release glucose through glycogenolysis, for it contains very limited amounts of glycogen [8].

One important function of the kidney is the reabsorption of glucose from glomerular filtrate. Glucose is freely filtered by the glomeruli. Approximately $180 \mathrm{~L}$ of plasma and around $180 \mathrm{~g}$ of glucose are filtered by the kidney every day. Under normal conditions, more than $99 \%$ of the filtered glucose is reabsorbed into the circulation, and the urine is free of glucose [9]. Sodium-glucose cotransporters (SGLTs) in the proximal convoluted tubule are the transporters responsible for kidney glucose reabsorption. There are six identified members of the SGLT family (SGLT1-6) [10]. In the kidney, the transport of glucose is mediated primarily by SGLT1 and SGLT2. Of these two transporters, SGLT1 plays important roles in glucose absorption/reabsorption and is mainly localized on the luminal side of the small intestinal epithelial cells and segment 3 (S3) of the renal proximal tubules. SGLT2 is a high-capacity, low-affinity glucose transporter that is predominantly located in the brush border membrane of the S1 and S2 segments of the renal proximal tubule and is responsible for reabsorption of more than $90 \%$ of the filtered glucose in the kidney [11]. The proximal tubule cells of the kidney actively contribute to glucose homeostasis by reabsorbing glucose through the transporter SGLT2.

In patients with diabetes, the blood glucose levels increase and exceed the transport maximum $\left(\mathrm{T}_{\mathrm{m}}\right)$ glucose level at a threshold of approximately $200 \mathrm{mg} / \mathrm{dL}$. It is reported that in diabetes, expression of the SGLT2 transporter gene is upregulated and the renal threshold increased, leading to increased glucose reabsorption from glomerular filtrate, thereby reducing urinary glucose excretion and worsening the hyperglycemic condition. In $d b / d b$ mice, genes involved in glucose metabolism such as SGLT2, phosphoenolpyruvate carboxykinase (Pck1), and glucose-6-phosphatase were selectively hypomethylated in the proximal tubules compared with control $\mathrm{db} / \mathrm{m}$ mice [12]. An aberrant increase in DNA methylation of the PPAR $\gamma$ coactivator-1 $\alpha$ (PGC-1 $\alpha$ ) gene promoter is observed in the skeletal muscles of diabetic patients.

In the kidney, hyperglycemia causes kidney injury by several mechanisms, including: (a) increased expression of transforming growth factor- $\beta$ (TGF- $\beta$ ) and overproduction of the mesangial matrix; (b) abnormal activation of protein kinase $C$, which causes mesangial expansion via TGF- $\beta$, vascular endothelial growth factor (VEGF), reactive oxygen species (ROS), and angiotensin II; (c) production of ROS; (d) activation of mitogen-activated protein kinase pathways [13]; (e) PPARs; and (f) overproduction of advanced glycation end products.
172

Kidney Dis 2017;3:171-180

DOI: $10.1159 / 000479874$
$\mathrm{Su} / \mathrm{Cao} / \mathrm{He} / \mathrm{Guan} / \mathrm{Ruan}$ 
Fig. 1. Insulin resistance plays a central role in dyslipidemia. In adipose tissue, insulin inhibits the hormone-sensitive lipase. Thus, insulin has an antilipolytic action. Insulin resistance (IR) increases lipolysis, enhancing free fatty acid (FFA) release from the adipose tissue to the circulation, which increases very-low-density lipoprotein (VLDL) production in the liver. In the presence of increased VLDL in the plasma in diabetic kidney disease patients, VLDL triglycerides (TG) can be exchanged for high-density lipoprotein (HDL) cholesterol and low-density lipoprotein (LDL) cholesterol under action by cholesteryl ester (CE) transfer protein (CETP), resulting in TG-rich, cholesterol-depleted HDL and LDL particles. The TG-rich HDL can undergo further hydrolysis, which leads to the dissociation and clearance of Apo A-1. The TG-rich LDL can be converted to small dense LDL (SD-LDL) after hydrolysis.

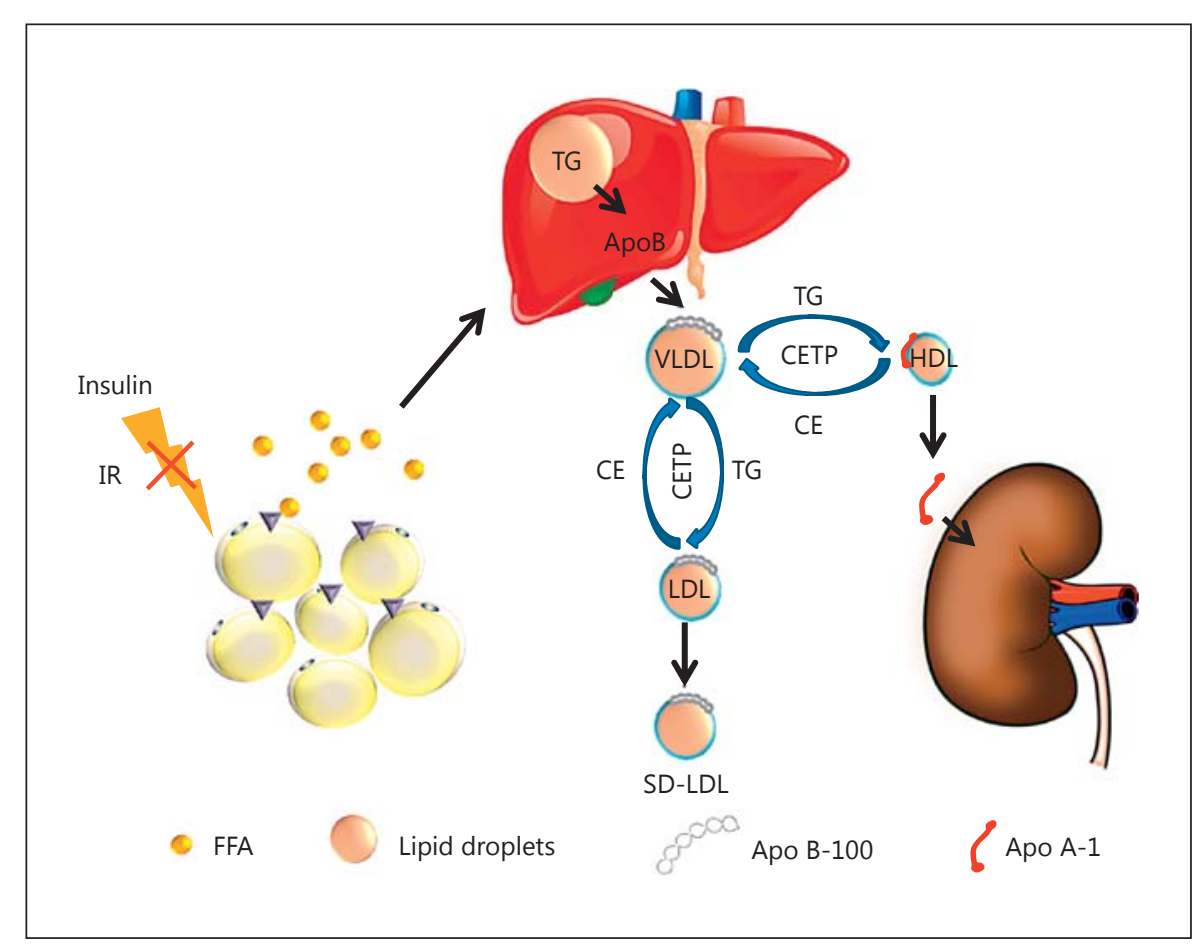

\section{Dyslipidemia and DKD}

Kimmelstiel and Wilson [14] first described the presence of lipid deposits in tubular epithelial cells (TECs) of diabetic kidneys in 1936. After that, a vast amount of evidence has shown that increased lipid accumulation in TECs would lead to lipotoxicity, which contributes to fibrosis [15]. Now there is growing evidence for the indispensable role of dyslipidemia, which is characterized by high plasma TG-rich lipoproteins, low plasma high-density lipoprotein cholesterol (HDL-C), and increased concentrations of small-sized and modified low-density lipoprotein particles (ox-LDL), in the progression of DKD patients $[16,17]$. Dyslipidemia in patients with type 2 diabetes (T2D) is a reversible risk factor for the progression of kidney disease. It is thus important to understand the roles of lipid disorder in the progression of DKD.

\section{DKD Is Usually Accompanied by Lipid Abnormalities}

Hyperglycemia is intrinsically linked to insulin resistance, which facilitates hyperlipidemia and contributes to renal disease pathogenesis in diabetic animals [18]. Excess carbohydrates can be converted by lipogenesis into free fatty acids (FAs) and TG by activation of acetyl-CoA carboxylase (ACC), FA synthase (FAS), or stearoyl-CoA desaturase-1. DKD is characterized by tubular epithelial lipid accumulation [14] and decreased FA oxidation (FAO). Heavy lipid deposition and increased lipid droplet accumulation are observed in DKD patients [19]. Insulin resistance increases FA lipolysis and release from adipose tissue into the circulation by activation of hormone-sensitive lipase, which is normally inhibited by insulin. As a consequence, very low-density lipoprotein (VLDL) synthesis and secretion from the liver are also increased. Increased levels of VLDL TG in the presence of cholesteryl ester transfer protein can promote the transfer of TG into LDL or HDL in exchange for LDL cholesteryl ester. The TG-rich LDL can undergo hydrolysis by hepatic lipase or lipoprotein lipase, which leads to a small dense LDL particle. The TG-rich HDL particle loses its normal function and can undergo further hydrolysis, which leads to the dissociation of Apo A-I from the HDL particle and low levels of HDL (Fig. 1). It has also been demonstrated that the LDL-C level is increased and a high level of LDL-C has been found to predict the development of microalbuminuria in type 1 diabetes (T1D). Toxic lipids such as lysophosphatidylcholine, ceramides, and free cholesterol may also accumulate in the tissues under insulin resistance. These results suggest that insulin resistance can cause both hyperglycemia and hyperlipidemia, but the crosstalk between lipid and glucose metabolism remains unclear. 
The Lipid Abnormalities Contribute to DKD

Dyslipidemia is observed in most DKD patients. The "lipid nephrotoxicity hypothesis" was first advocated by Moorhead et al. [20] to describe the effect of dyslipidemia on decreased kidney function in 1982, and updated by Ruan et al. [21] in 2009. As a consequence, dyslipidemia causes podocyte apoptosis and enhances macrophage infiltration and excessive extracellular matrix production in the glomeruli under diabetic conditions, contributing to the development of DKD. Furthermore, lipids can be accumulated in the kidney and directly cause kidney injury by increasing uptake via lipoprotein receptors/transporters, increasing lipogenesis or reducing efflux and consumption (oxidation). High cholesterol absorption and low cholesterol efflux in the kidney have been shown in different models of T1D [20] and T2D [21].

Enhanced lipogenic gene activation contributes to glomerular and tubular lipid deposits. Sterol regulatory element-binding proteins (SREBPs) are key regulators of lipid synthesis that play a central role in renal lipid accumulation in diabetic nephropathy [22]. SREBP1 and FAS expression is markedly increased in streptozotocin-induced diabetic nephropathic rat kidneys, resulting in TG and cholesterol accumulation in the kidney $[22,23]$. Induction of SREBP-1 may be related to increase in TGF- $\beta_{1}$ and accumulation of extracellular matrix [24]. Treatment of diabetic rats with insulin prevented the increased SREBP-1 and TG accumulation. SREBP-1a transgenic mice exhibited an increased renal TG content, increased expression of TGF- $\beta_{1}$ and mesangial expansion, glomerulosclerosis, and proteinuria [22]. Knockdown of SREBP-1 ameliorated the diabetic kidney injury and protected from cell dysfunction [22].

Evidence is emerging that reduced FAO is associated with lipid accumulation in DKD. An imbalance between circulating and cytosolic FA levels results in excessive intracellular accumulation of FAs. Carnitine palmitoyltransferase 1 is the rate-limiting enzyme in FAO. The PPARs and PGC-1 $\alpha$ are the key transcription factors that regulate the expression of proteins involved in FA uptake and oxidation. Activation of PPARa by fibrates reduces TG and elevates HDL levels, showing potential to reduce diabetic nephropathy $[25,26]$. Pretreatment with lowdose fenofibrate prevents the early onset of nephropathy in diabetic rats but is not effective in established diabetic rat nephropathy [27]. Acyl-CoA oxidase and liver-type FA-binding protein were reduced during DKD. PPAR activity is a potential cause of metabolic syndrome-related disorders. It has been proved that FAO is the key con- tributor to intracellular ATP levels in TECs [28]. FAO could be the key inciting factor for fibrosis development, and lipid accumulation occurs as a secondary consequence. Thus, reduced FAO might be a new mechanism in DKD.

CD36 is a transmembrane protein of the class B scavenger receptor family [25] functioning as an FA translocase which is expressed in several cell types including macrophages, microvascular endothelial cells, platelets, adipocytes, podocytes, and tubular cells. Long-chain FAs, particularly palmitate and stearate, enter cells mostly through CD36. CD36 expression is upregulated by hyperglycemia in DKD patients $[26,27]$ and is associated with increased uptake of ox-LDL and with tubular epithelial apoptosis, which in turn is associated with tubular degeneration and progression of DKD [27]. CD36 mediates TEC apoptosis and proinflammatory and profibrotic responses. TEC-specific overexpression of CD36 transgenic mice showed an increase in lipid accumulation in TECs. However, no higher susceptibility to diabetic kidney injury was observed. mRNA of the lipid droplet surface protein adipose differentiation-related protein (ADRP) was specifically upregulated 5.4-fold in 16 -week-old $d b / d b$ mouse kidneys [24], suggesting that the lipid droplet protein is involved in lipid accumulation in DKD kidneys. Podocytes are crucial for maintaining a normally functional glomerular filtration barrier. Lipotoxicity and lipid accumulation cause podocyte injury and apoptosis in DKD patients, which is a feature of $\mathrm{DKD}$, and podocytopenia is an independent predictor of DKD progression [29-32]. There are several mechanisms which contribute to podocyte loss in diabetes. The reduction in podocyte numbers contributes to the progression of DKD. Lipid droplet accumulation localized within the podocyte foot processes of patients with DKD was observed by Herman-Edelstein et al. [19]. This accumulation was associated with a downregulation of genes that encode proteins involved in cholesterol efflux (ABCA1, ABCG1, and APOE), an upregulation of LDL receptors, impaired FAO, and increased expression of angiopoietin-related protein 4 [19]. CD36 protein expression was markedly enhanced in podocytes treated with palmitic acid in a dose-dependent manner [33]. However, the exact role of CD36 in podocyte apoptosis in diabetic nephropathy with hyperlipidemia still needs to be elucidated. An in vitro experiment recently showed that high glucose induced VEGF and reduced podocyte viability via enhanced autophagy, which was thought to be a self-protective mechanism [34]. Overall, lipids and lipid-modulating proteins are 
Fig. 2. Glucose-lipid crosstalk in diabetic kidney disease. (1) SREBP-1 is the master regulator of lipid metabolism and can be upregulated by insulin, glucose, and liver $\mathrm{X}$ receptor (LXR). Farnesoid X receptor (FXR) can negatively regulate SREBP-1 expression in the kidney. In diabetic patients, hyperglycemia induces expression of SREBP-1, which enhances lipogenic gene expression and triglyceride (TG) synthesis and leads to excess lipid accumulation in the kidney. (2) Activation of AMP-activated protein kinase (AMPK) suppresses the transcription of SREBP-1 and prevents renal lipotoxicity. Activated AMPK can also participate in the glucose-lipid crosstalk through the $\mathrm{p}$-AMPK/Sirt-1/PGC-1a pathway or through suppression of mTOR. Drugs such as metformin can improve the lipid profile beyond its glucose-lowering effect. FA(S), fatty acid (synthase); ACC, acetyl-CoA carboxylase.

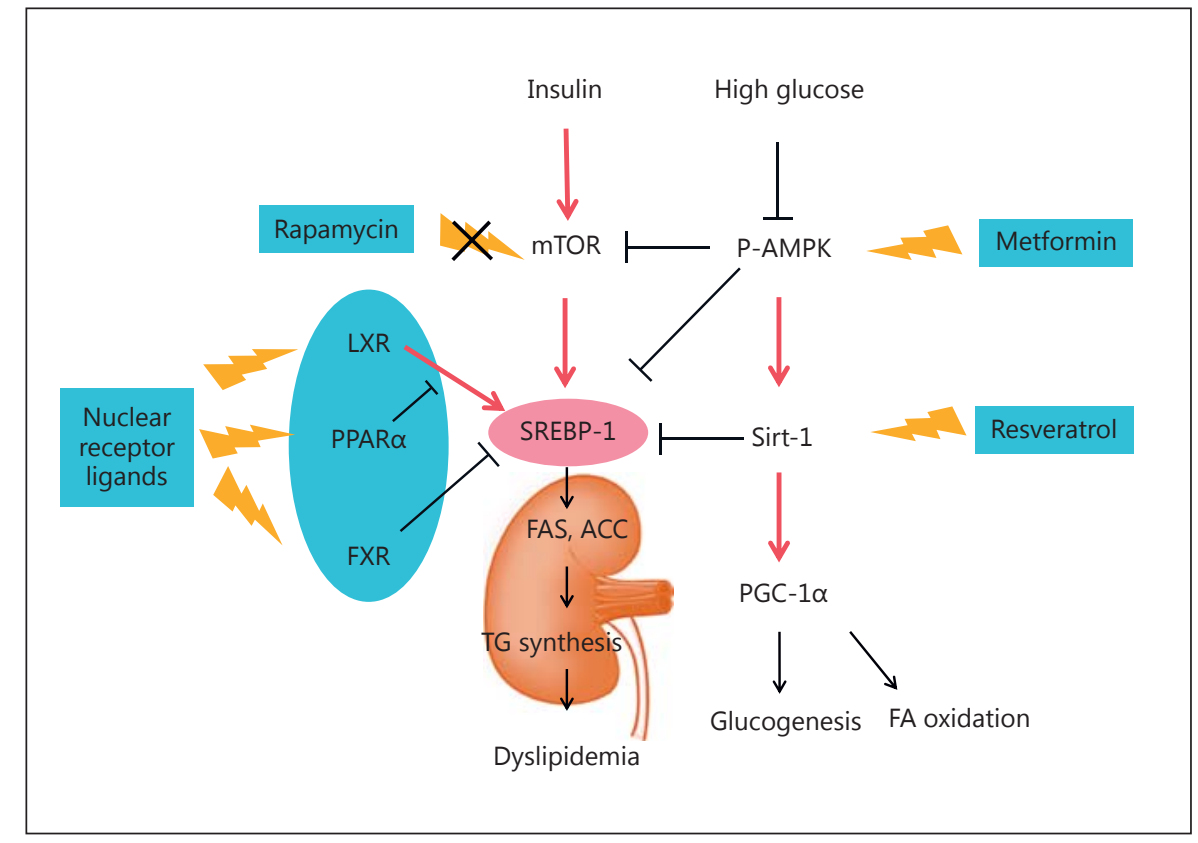

key determinants of podocyte biology and together contribute to the pathogenesis of glomerular diseases during DKD.

\section{Metabolic Dysregulation in DKD: Glucose-Lipid Crosstalk}

Metabolic dysregulation including hyperglycemia and hyperlipidemia initiates DKD. The alterations of glucose and lipid metabolism usually affect each other. It is well known that T2D is a metabolic disorder characterized by both hyperglycemia and dyslipidemia. Glucose-lowering drugs that control hyperglycemia usually also have an effect on lipid metabolism. Metformin lowers glucose while improving the lipid profile [35]. Also, a recent glucagonlike peptide-1 receptor agonist has proven efficacious in the glycemic control of T2D coupled with insulin-independent metabolic benefits for dyslipidemia [36]. The above-mentioned phenomena suggest that crosstalk between glucose and lipid metabolism exists during DKD. In this part, we will discuss the possible crosstalk between glucose and lipid metabolism from molecular networks and regulation pathways (Fig. 2).

\section{AMP-Activated Protein Kinase-Related Pathway}

AMP-activated protein kinase (AMPK) is an energy sensor that is activated by decreases in the cellular en- ergy state, as reflected in the AMP/ATP ratio [37]. Metformin can phosphorylate and activate AMPK through liver kinase B1. Activation of AMPK can suppress the transcription of several key lipogenic regulators in the liver, such as SREBP-1, ACC, and HMG-CoA reductase $[38,39]$. First, downregulation of SREBP-1 leads to a reduction in TG synthesis. Second, the inhibition of ACC enzyme activity also causes a reduction in FA and VLDL synthesis from the liver. Third, the suppression of HMGCoA reduces its capabilities of cholesterol synthesis. Metformin is reported to reduce TG by about $10 \%$ and LDL-C by $10-15 \%$, and to increase HDL-C up to $7 \%$. Therefore, metformin, through AMPK, improves the lipid profile beyond its glucose-lowering effect. The AMPK/silent information regulator T1 (SIRT1)/PGC$1 \alpha$ axis also participates in the crosstalk between lipid and glucose metabolism. Resveratrol is a natural plant polyphenol which activates SIRT1, and was reported to prevent renal lipotoxicity and to inhibit mesangial cell glycotoxicity in T2D mainly through the AMPK/SIRT1/ PGC- $1 a$ axis in a $d b / d b$ mouse model [40]. Activation of SIRT1 promotes gluconeogenesis and FAO through PGC-1 $\alpha[41,42]$.

SREBP-1 Serves as a Mediator for the Crosstalk between Glucose and Lipid Metabolism

In models of $\mathrm{DKD}$, an increased renal expression of SREBP has been observed [22, 24, 32]. SREBPs are tran- 
scription factors well-known for their important roles in lipid homeostasis; the three isoforms are SREBP-1a, -1c, and -2 . The inactive/precursor form of SREBP resides in the endoplasmic reticulum membrane. When activated, SREBP cleavage-activating protein binds and escorts SREBP from the endoplasmic reticulum to the Golgi complex and undergoes sequential proteolytic processing. Site 1 protease and site 2 protease are two proteases that could release the mature form of SREBP. Then, the active N-terminal domain of SREBP1 is translocated to the nucleus and the transcript regulates downstream target genes [43]. Transcriptional activation of SREBP-1 can also be upregulated by insulin [44], glucose [45], and LXR [46]. High-glucose-induced SREBP-1 was mediated by AMPK in T1D rats [47]. Uttarwar et al. [48] showed that in primary rat mesangial cells, high-glucose $(30 \mathrm{mM})$ treatment within $1 \mathrm{~h}$ could transcriptionally activate SREBP-1, which works through EGFR-PI3K-Akt signaling. Their data further showed that SREBP-1 could bind to the promoter region and directly regulates TGF- $\beta_{1}$ by glucose stimulation, mediating profibrogenic responses. In cultured renal tubular cells, high-glucose media could induce expression of SREBP-1 mRNA and protein levels, resulting in an enhanced cellular TG content through induction of FAS and ACC $[22,49]$. The relationship between SREBP-1 and an activated PI3K/Akt pathway has been confirmed in diabetic rats and in in vitro-cultured HKC cells [50]. Recently, mTOR was revealed to be involved in diabetic renal lipogenesis and could be a potential target for the treatment of DKD through regulation of SREBP-1 [51]. How mTORC1 regulates SREBP is still not fully understood, but it might be by controlling the nuclear entry of lipin-1, a phosphatidic acid phosphatase [52].

\section{Metabolic Nuclear Receptors}

Metabolic nuclear receptors consist of a group of nuclear hormone receptor transcription factors that play central roles in regulating metabolic balance. The metabolic nuclear receptors, including PPARs, LXRs, and FXRs, play important roles in regulating the crosstalk between glucose and lipid metabolism during DKD. Apart from the above-mentioned nuclear receptors, the other nuclear receptors, such as estrogen receptors, might also be involved in $\mathrm{DKD}$, based on the observation that $\mathrm{DKD}$ progresses faster in males than in females [53].

Peroxisome Proliferator-Activated Receptors

PPARs, including PPAR $\alpha$ (NR1C1), PPAR $\beta / \delta$ (NR1C2), and PPAR $\gamma$ (NR1C3), are key transcription factors in regulating glucose and lipid metabolism. PPARa is highly ex- pressed in the liver, kidney, and skeletal muscles, and is closely correlated with FAO [54]. In the kidney, PPARa is abundantly expressed in the proximal tubules and medullary thick ascending limbs, with lower expression levels in the glomeruli [55]. PPARa binds to a specific peroxisome proliferator response element, thereby forming a heterodimer with RXRa, and controls a set of genes essential for FA $\beta$-oxidation and has an important role in renal metabolic control [56]. PPARa gene deficiency markedly accelerated nephropathy in diabetic mice [57]. A PPARa agonist such as fenofibrate improves insulin sensitivity, glucose control, and DKD $[25,58]$. The mechanism of fenofibrate's renoprotective effect on DKD may be related to increased phosphorylation of AMPK, activation of PGC-1 $\alpha$, and amelioration of lipotoxicity in the kidney [59]. While improving hyperglycemia, fenofibrate treatment increased HDL-C with little change in VLDL in $d b / d b$ mice.

PPAR $\gamma$ is the master regulator of differentiation and energy storage by adipocytes and plays an important role in improving insulin sensitivity, which made it a key target for the treatment of T2D. PPAR $\gamma$ ligands regulate systemic lipid and glucose homeostasis. The most representative PPAR ligands, thiazolidinediones, show a strong ability both to lower glucose levels and to improve lipid profiles in T2D. In the kidney, PPAR $\gamma$ is most abundant in the collecting duct [60]. Activation of PPAR $\gamma$ modulates the transcription of a number of insulin-responsive genes involved in the control of glucose and lipid metabolism. PPAR $\alpha$ and PPAR $\gamma$ modulate energy utilization in the kidney by regulating FAO [61]. Activation of PPARa can stimulate FA $\beta$-oxidation, which can reduce the lipid content, and exerts renoprotective effects.

\section{Farnesoid X Receptor (NR1H4)}

FXR is a bile acid-activated nuclear receptor that is highly expressed in the liver, intestine, adrenal glands, and kidney. FXR plays a key role in bile acid, lipid, and glucose homeostasis. FXR is abundantly expressed in the kidney and negatively modulates renal SREBP-1 expression. The FXR agonist obeticholic acid or GW4064 could decrease kidney TG levels, suggesting that FXR might play a pivotal role in diabetic renal injury [31]. FXR deficiency caused increased kidney neutral lipid accumulation and accelerated type 1 diabetic nephropathy partly through enhanced SREBPs [62]. In contrast, treatment with the FXR agonist INT-747 significantly decreased total plasma cholesterol and LDL-C levels, and improved renal injury in a diabetic mouse model by modulating renal lipid metabolism and macrophage infiltration and by negatively modulating the renal expression of SREBPs [62].
176

Kidney Dis 2017;3:171-180

DOI: $10.1159 / 000479874$
$\mathrm{Su} / \mathrm{Cao} / \mathrm{He} / \mathrm{Guan} / \mathrm{Ruan}$ 
Liver X Receptors (NR1H3, NR1H2)

LXRs include two isoforms, namely, LXR $\alpha$ and LXR $\beta$. LXRa is highly expressed in lipid-metabolizing organs such as the liver, kidney, intestine, and adipose tissue [63], while LXR $\beta$ is more ubiquitously expressed [64]. The LXR agonist T0901317 could increase cholesterol efflux via activating ABCA1. Macrophage-specific activation of LXR markedly ameliorated hyperlipidemichyperglycemic nephropathy through the upregulation of genes related to cholesterol efflux and by suppressing glycated or acetylated LDL-induced cytokines and ROS [65]. However, applications of the LXR agonist have now been limited due to steatotic side effects.

\section{Therapeutic Strategy for DKD}

Diabetes treatment for patients with $\mathrm{DKD}$ is challenging. The progressive decline in renal function impairs the clearance and metabolism of antidiabetic drugs and insulin. Currently, there are no efficient therapies for slowing the progression of DKD. Evidence has shown that metabolic control could reduce the risk or slow the progression of DKD [66]. The landmark DCCT (Diabetes Control and Complications Trial)/EDIC (Epidemiology of Diabetes Interventions and Complications) research group showed a tight relationship between glucose control and complications and the treatment of T1D $[67,68]$. For most patients with T2D, metformin is recommended as an initial therapy [66]. As glycemic control worsens, other antidiabetic drugs with different mechanisms of action are added to metformin. The use of metformin in $\mathrm{DKD}$ is more complicated according to different degrees.

\section{SGLT2 Inhibitors: Pros and Cons}

The concept of using SGLT inhibitors as T2D targets is not new [11]. Phlorizin is a nonspecific SGLT1/2 inhibitor; already in 1835 it was found to increase glycosuria and reduce hyperglycemia and to normalize insulin sensitivity in T2D mice [69]. However, the nonselectivity for SGLT1 stopped this drug from being developed for clinical use, since SGLT1 also localizes at the intestinal brush border, which is responsible for absorption of dietary glucose [10]. A bunch of highly specific SGLT2 inhibitors are currently being developed by several drug companies. The benefits of SGLT2 inhibitors compared with other glucose-lowering drugs include the following: (1) the action of SGLT2 inhibitors should be independent of insulin action or pancreatic $\beta$-cell function; (2) they ameliorate hyperglycemia without causing weight gain or

Glucose-Lipid Crosstalk in DKD hypoglycemia, which is a common side effect of other T2D drugs [70, 71]; and (3) they lower blood pressure. Treatment with SGLT2 inhibitors has demonstrated improvements in $\mathrm{HbA}_{1 \mathrm{c}}$, reductions in body weight, and modest lowering of blood pressure. Empagliflozin is a potent and well-studied SGLT-2 inhibitor that was approved by the FDA in 2014 [72]. A recent study showed that empagliflozin could be combined with metformin to provide dual medications in a once-daily tablet to improve longterm $\mathrm{HgA}_{1 \mathrm{c}}$ when compared to metformin alone. Luseogliflozin is a highly selective and potent SGLT2 inhibitor [73] that has been approved for use as monotherapy or in combination with other antidiabetic drugs [74]. A single dose of luseogliflozin significantly increased 24-h urinary glucose excretion and significantly decreased fasting blood glucose and 2-h postprandial plasma glucose in patients with normal-to-moderately reduced renal function but not severely reduced renal function. The ability of the SGLT2 inhibitor to reduce plasma glucose levels is associated with the GFR [75].

\section{Hyperlipidemia: A New Therapeutic Target for DKD?}

Clinical studies have demonstrated that lipid-lowering therapy (with statins and fibrates) shows a protective effect on renal function by improving albuminuria and the estimated GFR [76]. Statins, which target cholesterol synthesis, may improve the estimated GFR through downregulation of small GTP-binding proteins and its downstream Rho/Rho-kinase proteins. Statins may decrease albuminuria, but they cannot stop the progression of $\mathrm{DKD}$, indicating that other pathways besides cholesterol synthesis contribute to cholesterol accumulation in the kidneys of DKD patients. Moreover, the effects of statins vary with the stage of chronic kidney disease (CKD). Moderate- to high-quality evidence indicates that statins reduce all-cause mortality, cardiovascular mortality, and cardiovascular events. Thus, other therapeutic strategies for reducing cholesterol accumulation in peripheral organs, especially the kidney, need further investigation. Proprotein convertase subtilisin/kexin type 9 (PCSK9) is a serine protease that is produced predominantly in the liver. Through binding to hepatic LDL receptors and promoting their degradation, PCSK9 downregulates plasma levels of LDL-C [77, 78]. PCSK9 is elevated in CKD and proteinuria. Alirocumab [79] and evolocumab [80] are fully human monoclonal antibodies against PCSK9. They reduce LDL-C by up to $65 \%$ and have been well tolerated in randomized, placebo-controlled, phase II clinical trials up to 1 year in over 3,000 hypercholesterolemic patients [81-85]. In a phase III clinical trial, $420 \mathrm{mg}$ of evolocum-

Kidney Dis 2017;3:171-180 177 
ab every 4 weeks for 52 weeks resulted in a relative reduction in LDL-C levels of 57\% [86].

\section{Metabolic Nuclear Receptors: Great Promise?}

Now clinical trials and animal studies have taken more interest in the function of nuclear hormone receptors in protection against kidney disease. Fenofibrate is a classic PPARa agonist. It has been demonstrated in multiple animal studies that fenofibrate reduces albuminuria in patients with diabetes $[25,61,87]$. In addition to reducing albuminuria, fenofibrate has been reported to ameliorate hyperglycemia [88, 89]. Acute fibrate-induced creatinine elevation in T2D with relatively preserved renal function may confer longer-term renoprotective effects. Recent studies reported that FXR negatively modulates renal SREBP-1 expression [31, 90] and its activation prevents type 1 diabetic nephropathy [62]. Treatment with the FXR agonist INT-747 improves renal lipid metabolism through negative regulation of SREBP-1 while decreasing proteinuria, glomerulosclerosis, and tubulointerstitial fibrosis [62]. Moreover, activation of FXR by the synthetic agonist GW4064 or by adenovirus-mediated FXR hepatic overexpression improves both hyperglycemia and hyperlipidemia in $d b / d b$ mice [91]. Thus, FXR indicates that is has therapeutic potential for DKD.

\section{Conclusion}

As the number of DKD patients continues to increase worldwide - and, most importantly, the current glucose control strategy is not able to fully prevent $\mathrm{DKD}$ develop- ment - novel effective and long-lasting therapies for DKD are urgently needed. In this review, we highlighted the molecular crosstalk between dyslipidemia and hyperglycemia in progression of DKD. The roles of AMPK, SREBP-1, and some metabolic hormone receptors including LXR, FXR, and PPARs on the crosstalk between lipid and glucose homeostasis were discussed. Finally, we provided an update on recent drug studies based on due regulation of lipid and glucose in DKD. Lipoprotein may become a new target in CKD patients, especially patients with proteinuric or nephrotic syndrome. Recent advances in the understanding of metabolic nuclear receptors have opened a window for the development of novel drugs. Since SREBP-1 or SREBP-2 inhibitors are still unavailable, metabolic nuclear receptors may be potential candidates for the development of new drugs with which both glucose and lipid disorders can be controlled.

\section{Acknowledgements}

This study was supported by grant 2012CB517504 from the Ministry of Science and Technology and grant 81500538 from the National Natural Science Foundation. The project was also funded by the China Postdoctoral Science Foundation (2015M570724), Shenzhen Peacock Program (KQTD20140630100746562), and Shenzhen Research Projects (JCYJ20140509172719310, CXZZ20150601140615135, and JCYJ20160307160819191).

\section{Conflict of Interest Statement}

The authors declare no competing interests.

\section{References}

1 Reidy K, Kang HM, Hostetter T, Susztak K: Molecular mechanisms of diabetic kidney disease. J Clin Invest 2014;124:2333-2340.

2 Gregg EW, Li Y, Wang J, Burrows NR, Ali MK, Rolka D, et al: Changes in diabetes-related complications in the United States, 19902010. N Engl J Med 2014;370:1514-1523.

3 Collins AJ, Foley RN, Chavers B, Gilbertson D, Herzog C, Johansen K, et al: United States Renal Data System 2011 Annual Data Report: atlas of chronic kidney disease and end-stage renal disease in the United States. Am J Kidney Dis 2012;59(suppl 1):A7, e1-e420.

4 Satchell SC: The glomerular endothelium emerges as a key player in diabetic nephropathy. Kidney Int 2012;82:949-951.
5 Stadler K, Goldberg IJ, Susztak K: The evolving understanding of the contribution of lipid metabolism to diabetic kidney disease. Curr Diab Rep 2015;15:40.

6 Gosmanov AR, Gosmanova EO: Long-term renal outcomes of patients with type 1 diabetes mellitus and microalbuminuria: an analysis of the DCCT/EDIC cohort. Arch Intern Med 2011;171:1596; author reply 1597.

7 Francis GA, Fayard E, Picard F, Auwerx J: Nuclear receptors and the control of metabolism. Annu Rev Physiol 2003;65:261-311.

8 Stumvoll M, Meyer C, Mitrakou A, Nadkarni V, Gerich JE: Renal glucose production and utilization: new aspects in humans. Diabetologia 1997;40:749-757.

9 Mather A, Pollock C: Glucose handling by the kidney. Kidney Int Suppl 2011;120:S1-S6.
10 Wright EM, Hirayama BA, Loo DF: Active sugar transport in health and disease. J Intern Med 2007;261:32-43.

11 Wright EM, Turk E: The sodium/glucose cotransport family SLC5. Pflugers Arch 2004; 447:510-518.

12 Marumo T, Yagi S, Kawarazaki W, Nishimoto M, Ayuzawa N, Watanabe A, et al: Diabetes induces aberrant DNA methylation in the proximal tubules of the kidney. J Am Soc Nephrol 2015;26:2388-2397.

13 Lander HM, Tauras JM, Ogiste JS, Hori O, Moss RA, Schmidt AM: Activation of the receptor for advanced glycation end products triggers a $\mathrm{p} 21^{\text {ras }}$-dependent mitogen-activated protein kinase pathway regulated by oxidant stress. J Biol Chem 1997;272:1781017814. 
14 Kimmelstiel P, Wilson C: Intercapillary lesions in the glomeruli of the kidney. Am J Pathol 1936;12:83-98.7.

15 Murea M, Freedman BI, Parks JS, Antinozzi PA, Elbein SC, Ma L: Lipotoxicity in diabetic nephropathy: the potential role of fatty acid oxidation. Clin J Am Soc Nephrol 2010;5: 2373-2379.

16 Hirano T: Lipoprotein abnormalities in diabetic nephropathy. Kidney Int Suppl 1999; 71:S22-S24.

17 Leiter LA: The prevention of diabetic microvascular complications of diabetes: is there a role for lipid lowering? Diabetes Res Clin Pract 2005;68(suppl 2):S3-S14.

18 Dronavalli S, Duka I, Bakris GL: The pathogenesis of diabetic nephropathy. Nat Clin Pract Endocrinol Metab 2008;4:444-452.

19 Herman-Edelstein M, Scherzer P, Tobar A, Levi M, Gafter U: Altered renal lipid metabolism and renal lipid accumulation in human diabetic nephropathy. J Lipid Res 2014;55: 561-572.

20 Moorhead JF, Chan MK, El-Nahas M, Varghese Z: Lipid nephrotoxicity in chronic progressive glomerular and tubulo-interstitial disease. Lancet 1982;2:1309-1311.

21 Ruan XZ, Varghese Z, Moorhead JF: An update on the lipid nephrotoxicity hypothesis. Nat Rev Nephrol 2009;5:713-721.

22 Sun L, Halaihel N, Zhang W, Rogers T, Levi M: Role of sterol regulatory element-binding protein 1 in regulation of renal lipid metabolism and glomerulosclerosis in diabetes mellitus. J Biol Chem 2002;277:18919-18927.

23 Ishigaki N, Yamamoto T, Shimizu Y, Kobayashi $\mathrm{K}$, Yatoh S, Sone H, et al: Involvement of glomerular SREBP-1c in diabetic nephropathy. Biochem Biophys Res Commun 2007; 364:502-508.

24 Jun $\mathrm{H}$, Song Z, Chen W, Zanhua R, Yonghong $S$, Shuxia L, et al: In vivo and in vitro effects of SREBP-1 on diabetic renal tubular lipid accumulation and RNAi-mediated gene silencing study. Histochem Cell Biol 2009;131:327345.

25 Park CW, Zhang Y, Zhang X, Wu J, Chen L, Cha DR, et al: PPARa agonist fenofibrate improves diabetic nephropathy in $d b / d b$ mice. Kidney Int 2006;69:1511-1517.

26 Ansquer JC, Foucher C, Rattier S, Taskinen MR, Steiner G; DAIS Investigators: Fenofibrate reduces progression to microalbuminuria over 3 years in a placebo-controlled study in type 2 diabetes: results from the Diabetes Atherosclerosis Intervention Study (DAIS). Am J Kidney Dis 2005;45:485-493.

27 Kadian S, Mahadevan N, Balakumar P: Differential effects of low-dose fenofibrate treatment in diabetic rats with early onset nephropathy and established nephropathy. Eur J Pharmacol 2013;698:388-396.

28 Kang HM, Ahn SH, Choi P, Ko YA, Han SH, Chinga $F$, et al: Defective fatty acid oxidation in renal tubular epithelial cells has a key role in kidney fibrosis development. Nat Med 2015;21:37-46.
29 Pagtalunan ME, Miller PL, Jumping-Eagle S, Nelson RG, Myers BD, Rennke HG, et al: Podocyte loss and progressive glomerular injury in type II diabetes. J Clin Invest 1997;99: 342-348.

30 Proctor G, Jiang T, Iwahashi M, Wang Z, Li J, Levi M: Regulation of renal fatty acid and cholesterol metabolism, inflammation, and fibrosis in Akita and OVE26 mice with type 1 diabetes. Diabetes 2006;55:2502-2509.

31 Jiang T, Wang XX, Scherzer P, Wilson P, Tallman J, Takahashi $\mathrm{H}$, et al: Farnesoid X receptor modulates renal lipid metabolism, fibrosis, and diabetic nephropathy. Diabetes 2007; 56:2485-2493.

32 Wang Z, Jiang T, Li J, Proctor G, McManaman JL, Lucia S, et al: Regulation of renal lipid metabolism, lipid accumulation, and glomerulosclerosis in $\mathrm{FVB}^{d b / d b}$ mice with type 2 diabetes. Diabetes 2005;54:2328-2335.

33 Hua W, Huang HZ, Tan LT, Wan JM, Gui HB, Zhao L, et al: CD36 mediated fatty acidinduced podocyte apoptosis via oxidative stress. PLoS One 2015;10:e127507.

34 Miaomiao W, Chunhua L, Xiaochen Z, Xiaoniao C, Hongli L, Zhuo Y: Autophagy is involved in regulating VEGF during high-glucose-induced podocyte injury. Mol Biosyst 2016;12:2202-2212.

35 Hundal RS, Krssak M, Dufour S, Laurent D, Lebon V, Chandramouli V, et al: Mechanism by which metformin reduces glucose production in type 2 diabetes. Diabetes 2000;49: 2063-2069.

36 Marre M, Shaw J, Brändle M, Bebakar WM, Kamaruddin NA, Strand J, et al: Liraglutide, a once-daily human GLP-1 analogue, added to a sulphonylurea over 26 weeks produces greater improvements in glycaemic and weight control compared with adding rosiglitazone or placebo in subjects with type 2 diabetes (LEAD-1 SU). Diabet Med 2009;26: 268-278.

37 Kahn BB, Alquier T, Carling D, Hardie DG: AMP-activated protein kinase: ancient energy gauge provides clues to modern understanding of metabolism. Cell Metab 2005;1: $15-25$.

38 Wang W, Guo XH, Wu HH, Wang NH, Xu XS: Effect of fenofibrate and metformin on lipotoxicity in OLETF rat kidney (in Chinese). Beijing Da Xue Xue Bao 2006;38:170-175.

39 Li Y, Xu S, Mihaylova MM, Zheng B, Hou X, Jiang B, et al: AMPK phosphorylates and inhibits SREBP activity to attenuate hepatic steatosis and atherosclerosis in diet-induced insulin-resistant mice. Cell Metab 2011;13:376-388.

40 Kim MY, Lim JH, Youn HH, Hong YA, Yang KS, Park HS, et al: Resveratrol prevents renal lipotoxicity and inhibits mesangial cell glucotoxicity in a manner dependent on the AMPK-SIRT1-PGC1 $a$ axis in $d b / d b$ mice. Diabetologia 2013;56:204-217.

41 Rodgers JT, Lerin C, Haas W, Gygi SP, Spiegelman BM, Puigserver P: Nutrient control of glucose homeostasis through a complex of PGC$1 \alpha$ and SIRT1. Nature 2005;434:113-118.
42 Lagouge M, Argmann C, Gerhart-Hines Z, Meziane H, Lerin C, Daussin F, et al: Resveratrol improves mitochondrial function and protects against metabolic disease by activating SIRT1 and PGC-1a. Cell 2006;127:1109-1122.

43 Brown MS, Goldstein JL: A proteolytic pathway that controls the cholesterol content of membranes, cells, and blood. Proc Natl Acad Sci USA 1999;96:11041-11048.

44 Kim JB, Sarraf P, Wright M, Yao KM, Mueller E, Solanes G, et al: Nutritional and insulin regulation of fatty acid synthetase and leptin gene expression through ADD1/SREBP1. J Clin Invest 1998;101:1-9.

45 Hasty AH, Shimano H, Yahagi N, AmemiyaKudo M, Perrey S, Yoshikawa T, et al: Sterol regulatory element-binding protein- 1 is regulated by glucose at the transcriptional level. J Biol Chem 2000;275:31069-31077.

46 Repa JJ, Liang G, Ou J, Bashmakov Y, Lobaccaro JM, Shimomura I, et al: Regulation of mouse sterol regulatory element-binding protein-1c gene (SREBP-1c) by oxysterol receptors, LXR $\alpha$ and LXR $\beta$. Genes Dev 2000;14: 2819-2830.

47 Soetikno V, Sari FR, Sukumaran V, Lakshmanan AP, Harima M, Suzuki K, et al: Curcumin decreases renal triglyceride accumulation through AMPK-SREBP signaling pathway in streptozotocin-induced type 1 diabetic rats. J Nutr Biochem 2013;24:796-802.

48 Uttarwar L, Gao B, Ingram AJ, Krepinsky JC: SREBP-1 activation by glucose mediates TGF- $\beta$ upregulation in mesangial cells. Am J Physiol Renal Physiol 2012;302:F329-F341.

49 Hao J, Zhu L, Zhao S, Liu S, Liu Q, Duan H: PTEN ameliorates high glucose-induced lipid deposits through regulating SREBP-1/FASN/ ACC pathway in renal proximal tubular cells. Exp Cell Res 2011;317:1629-1639.

50 Hao J, Liu S, Zhao S, Liu Q, Lv X, Chen H, et al: PI3K/Akt pathway mediates high glucoseinduced lipogenesis and extracellular matrix accumulation in HKC cells through regulation of SREBP- 1 and TGF- $\beta 1$. Histochem Cell Biol 2011;135:173-181.

51 Hao J, Zhu L, Li F, Liu Q, Zhao X, Liu S, et al: Phospho-mTOR: a novel target in regulation of renal lipid metabolism abnormality of diabetes. Exp Cell Res 2013;319:2296-2306.

52 Peterson TR, Sengupta SS, Harris TE, Carmack AE, Kang SA, Balderas E, et al: mTOR complex 1 regulates lipin 1 localization to control the SREBP pathway. Cell 2011;146:408-420.

53 Sibley SD, Thomas W, de Boer I, Brunzell JD, Steffes MW: Gender and elevated albumin excretion in the Diabetes Control and Complications Trial/Epidemiology of Diabetes Interventions and Complications (DCCT/EDIC) cohort: role of central obesity. Am J Kidney Dis 2006;47:223-232.

54 Braissant O, Foufelle F, Scotto C, Dauça M, Wahli W: Differential expression of peroxisome proliferator-activated receptors (PPARs): tissue distribution of PPAR- $\alpha,-\beta$, and $-\gamma$ in the adult rat. Endocrinology 1996; 137:354-366. 
55 Guan Y, Zhang Y, Davis L, Breyer MD: Expression of peroxisome proliferator-activated receptors in urinary tract of rabbits and humans. Am J Physiol 1997;273(pt 2):F1013F1022.

56 Portilla D: Energy metabolism and cytotoxicity. Semin Nephrol 2003;23:432-438.

57 Park CW, Kim HW, Ko SH, Chung HW, Lim SW, Yang CW, et al: Accelerated diabetic nephropathy in mice lacking the peroxisome proliferator-activated receptor $\alpha$. Diabetes 2006;55:885-893.

58 Zhou Y, Kong X, Zhao P, Yang H, Chen L, Miao J, et al: Peroxisome proliferator-activated receptor- $\alpha$ is renoprotective in doxorubicin-induced glomerular injury. Kidney Int 2011;79:1302-1311.

59 Hong YA, Lim JH, Kim MY, Kim TW, Kim Y, Yang KS, et al: Fenofibrate improves renal lipotoxicity through activation of AMPKPGC- $1 a$ in $d b / d b$ mice. PLoS One 2014;9: e96147.

60 Guan Y, Hao C, Cha DR, Rao R, Lu W, Kohan $\mathrm{DE}$, et al: Thiazolidinediones expand body fluid volume through PPAR $\gamma$ stimulation of $\mathrm{ENaC}$-mediated renal salt absorption. Nat Med 2005;11:861-866.

$61 \mathrm{Wu}$ J, Chen L, Zhang D, Huo M, Zhang X, Pu $\mathrm{D}$, et al: Peroxisome proliferator-activated receptors and renal diseases. Front Biosci (Landmark Ed) 2009;14:995-1009.

62 Wang XX, Jiang T, Shen Y, Caldas Y, Miyazaki-Anzai S, Santamaria H, et al: Diabetic nephropathy is accelerated by farnesoid $\mathrm{X}$ receptor deficiency and inhibited by farnesoid $\mathrm{X}$ receptor activation in a type 1 diabetes model. Diabetes 2010;59:2916-2927.

63 Schultz JR, Tu H, Luk A, Repa JJ, Medina JC, $\mathrm{Li}$ L, et al: Role of LXRs in control of lipogenesis. Genes Dev 2000;14:2831-2838.

64 Repa JJ, Mangelsdorf DJ: The role of orphan nuclear receptors in the regulation of cholesterol homeostasis. Annu Rev Cell Dev Biol 2000;16:459-481.

65 Kiss E, Kränzlin B, Wagenblass K, Bonrouhi $M$, Thiery J, Gröne E, et al: Lipid droplet accumulation is associated with an increase in hyperglycemia-induced renal damage: prevention by liver $\mathrm{X}$ receptors. Am J Pathol 2013;182:727-741.

66 American Diabetes Association: Standards of Medical Care in Diabetes - 2016 Abridged for Primary Care Providers. Clin Diabetes 2016; 34:3-21.

67 Diabetes Control and Complications Trial Research Group, Nathan DM, Genuth S, Lachin J, Cleary P, Crofford O, Davis M, et al: The effect of intensive treatment of diabetes on the development and progression of longterm complications in insulin-dependent diabetes mellitus. N Engl J Med 1993;329:977986.

68 Nathan DM, Bayless M, Cleary P, Genuth S, Gubitosi-Klug R, Lachin JM, et al: Diabetes control and complications trial/epidemiology of diabetes interventions and complications study at 30 years: advances and contributions. Diabetes 2013;62:3976-3986.

69 Rossetti L, Smith D, Shulman GI, Papachristou D, DeFronzo RA: Correction of hyperglycemia with phlorizin normalizes tissue sensitivity to insulin in diabetic rats. J Clin Invest 1987;79:1510-1515.

70 Bays H: Sodium glucose co-transporter type 2 (SGLT2) inhibitors: targeting the kidney to improve glycemic control in diabetes mellitus. Diabetes Ther 2013;4:195-220.

71 Tahrani AA, Barnett AH, Bailey CJ: SGLT inhibitors in management of diabetes. Lancet Diabetes Endocrinol 2013;1:140-151.

72 Neumiller JJ: Empagliflozin: a new sodiumglucose co-transporter 2 (SGLT2) inhibitor for the treatment of type 2 diabetes. Drugs Context 2014;3:212262.

73 Jinnouchi $H$, Nozaki K, Watase $H$, Omiya $H$, Sakai S, Samukawa Y: Impact of reduced renal function on the glucose-lowering effects of luseogliflozin, a selective SGLT2 inhibitor, assessed by continuous glucose monitoring in Japanese patients with type 2 diabetes mellitus. Adv Ther 2016;33:460-479.

74 Seino Y, Sasaki T, Fukatsu A, Ubukata M, Sakai S, Samukawa Y: Efficacy and safety of luseogliflozin as monotherapy in Japanese patients with type 2 diabetes mellitus: a randomized, double-blind, placebo-controlled, phase 3 study. Curr Med Res Opin 2014;30:12451255.

75 Gerich JE: Role of the kidney in normal glucose homeostasis and in the hyperglycaemia of diabetes mellitus: therapeutic implications. Diabet Med 2010;27:136-142.

76 Rosario RF, Prabhakar S: Lipids and diabetic nephropathy. Curr Diab Rep 2006;6:455-462.

77 Lambert G, Sjouke B, Choque B, Kastelein JJ, Hovingh GK: The PCSK9 decade. J Lipid Res 2012;53:2515-2524.

78 Stein EA, Swergold GD: Potential of proprotein convertase subtilisin/kexin type 9 based therapeutics. Curr Atheroscler Rep 2013;15: 310.

79 Roth EM, McKenney JM, Hanotin C, Asset G, Stein EA: Atorvastatin with or without an antibody to PCSK9 in primary hypercholesterolemia. N Engl J Med 2012;367:1891-1900.

80 Nissen SE, Dent-Acosta RE, Rosenson RS, Stroes E, Sattar N, Preiss D, et al: Comparison of PCSK9 inhibitor evolocumab versus ezetimibe in statin-intolerant patients: design of the Goal Achievement after Utilizing an AntiPCSK9 Antibody in Statin-Intolerant Subjects 3 (GAUSS-3) Trial. Clin Cardiol 2016; 39:137-144.

81 Stein EA, Gipe D, Bergeron J, Gaudet D, Weiss R, Dufour R, et al: Effect of a monoclonal antibody to PCSK9, REGN727/ SAR236553, to reduce low-density lipoprotein cholesterol in patients with heterozygous familial hypercholesterolaemia on stable statin dose with or without ezetimibe therapy: a phase 2 randomised controlled trial. Lancet 2012;380:29-36.
82 Sullivan D, Olsson AG, Scott R, Kim JB, Xue A, Gebski V, et al: Effect of a monoclonal antibody to PCSK9 on low-density lipoprotein cholesterol levels in statin-intolerant patients: the GAUSS randomized trial. JAMA 2012; 308:2497-2506

83 McKenney JM, Koren MJ, Kereiakes DJ, Hanotin C, Ferrand AC, Stein EA: Safety and efficacy of a monoclonal antibody to proprotein convertase subtilisin/kexin type 9 serine protease, SAR236553/REGN727, in patients with primary hypercholesterolemia receiving ongoing stable atorvastatin therapy. J Am Coll Cardiol 2012;59:2344-2353.

84 Giugliano RP, Desai NR, Kohli P, Rogers WJ, Somaratne R, Huang F, et al; LAPLACE-TIMI 57 Investigators: Efficacy, safety, and tolerability of a monoclonal antibody to proprotein convertase subtilisin/kexin type 9 in combination with a statin in patients with hypercholesterolaemia (LAPLACE-TIMI 57): a randomised, placebo-controlled, dose-ranging, phase 2 study. Lancet 2012;380:2007-2017.

85 Raal F, Scott R, Somaratne R, Bridges I, Li G, Wasserman SM, et al: Low-density lipoprotein cholesterol-lowering effects of AMG 145, a monoclonal antibody to proprotein convertase subtilisin/kexin type 9 serine protease in patients with heterozygous familial hypercholesterolemia: the Reduction of LDL-C with PCSK9 Inhibition in Heterozygous Familial Hypercholesterolemia Disorder (RUTHERFORD) randomized trial. Circulation 2012;126:2408-2417.

86 Blom DJ, Hala T, Bolognese M, Lillestol MJ, Toth PD, Burgess L, et al; DESCARTES Investigators: A 52-week placebo-controlled trial of evolocumab in hyperlipidemia. N Engl J Med 2014;370:1809-1819.

87 Gabbi C, Kong X, Suzuki H, Kim HJ, Gao M, Jia X, et al: Central diabetes insipidus associated with impaired renal aquaporin-1 expression in mice lacking liver $\mathrm{X}$ receptor $\beta$. Proc Natl Acad Sci USA 2012;109:3030-3034.

88 Xie W, Nie Y, Du L, Zhang Y, Cai G: Preventive effects of fenofibrate on insulin resistance, hyperglycaemia, visceral fat accumulation in NIH mice induced by small-dose streptozotocin and lard. Pharmacol Res 2007; 55:392-399.

89 Liu J, Lu R, Wang Y, Hu Y, Jia Y, Yang N, et al: PPAR $a$ agonist fenofibrate reduced the secreting load of $\beta$-cells in hypertriglyceridemia patients with normal glucose tolerance. PPAR Res 2016;2016:6232036.

90 Wang XX, Jiang T, Shen Y, Adorini L, Pruzanski M, Gonzalez FJ, et al: The farnesoid X receptor modulates renal lipid metabolism and diet-induced renal inflammation, fibrosis, and proteinuria. Am J Physiol Renal Physiol 2009;297:F1587-F1596.

91 Zhang Y, Lee FY, Barrera G, Lee H, Vales C, Gonzalez FJ, et al: Activation of the nuclear receptor FXR improves hyperglycemia and hyperlipidemia in diabetic mice. Proc Natl Acad Sci USA 2006;103:1006-1011. 